\title{
Two different approaches to handle landmark location uncertainty in skull-face overlay: coevolution vs fuzzy landmarks
}

\author{
Óscar Ibáñez ${ }^{1}$ Óscar Cordón ${ }^{1}$ Sergio Damas ${ }^{1}$ \\ ${ }^{1}$ European Centre for Soft Computing
}

\begin{abstract}
Craniofacial superimposition is a forensic process where photographs or video shots of a missing person are compared with the skull that is found. By projecting both photographs on top of each other (or, even better, matching a scanned three-dimensional skull model against the face photo/video shot), the forensic anthropologist can try to establish whether that is the same person. The whole process is influenced by inherent uncertainty mainly because two objects of different nature (a skull and a face) are involved. In this paper, we propose a novel approach, a cooperative coevolutionary algorithm, to deal with the use of imprecise cephalometric landmarks in the skull-face overlay process. Following this approach we are able to look for both the best transformation parameters and the best landmark locations at the same time. Coevolutionary skull-face overlay results are compared with our previous fuzzy-evolutionary automatic method over six skull-face overlay problem instances corresponding to three real-world cases solved by the Physical Anthropology lab at the University of Granada (Spain). Promising results have been achieved though the robustness of the method should be improved.
\end{abstract}

Keywords: Forensic identification, craniofacial superimposition, skull-face overlay, coevolution, cooperative coevolutionary algorithm, fuzzy landmarks, evolutionary algorithms.

\section{Introduction}

Craniofacial superimposition (CS) $[1,2,3]$ is a forensic process where photographs or video shots of a missing person are compared with the skull that is found. By projecting both photographs on top of each other (or, even better, matching a scanned three-dimensional skull model against the face photo/series of video shots), the forensic anthropologist can try to establish whether that is the same person. This skull-face overlay (SFO) process is usually done by bringing to match some corresponding anthropometrical landmarks on the skull and the face.

SFO is known to be one of the most time consuming tasks for the forensic experts [4] (it takes up to
24 hours in many real-world situations).

As it is explained in [5], we have developed an automatic method that overlays a $3 \mathrm{D}$ model of the skull over a 2D photograph of the face of the missing person. With that aim, we studied and experimented with different evolutionary approaches in [5], where CMA-ES demonstrated to be the most accurate and robust approach.

The SFO process is influenced by inherent uncertainty since two objects of different nature are involved (a skull and a face) [6]. In [7] we studied in detail the sources of uncertainty related to the SFO task and proposed the use of imprecise landmarks to overcome most of the limitations associated with them. Using imprecise landmarks, forensic anthropologists are able to properly deal with two different problems. On the one hand, the difficult task of locating anthropometric landmarks invariantly. On the other hand, the identification of a big enough set of non coplanar landmarks. These imprecise landmarks were modeled following the fuzzy set theorybased approach and their use led to a really important performance improvement of our automatic method.

However, the fuzzy-evolutionary approach relies on more computational operations due to the fact that distances between a crisp point and a fuzzy set of points must be computed. Hence, the run time required by the algorithm increased. In particular, the crisp landmark approach ranged in 10-20 seconds per run while the fuzzy landmark approach ranged in 2-4 minutes.

With the aim of decreasing the run time needed without loosing accuracy, we propose a new evolutionary approach based on a coevolutionary algorithm (CEA) [8]. It also takes advantage of imprecise landmarks but, unlike the fuzzy approach where distances between a fuzzy set and a crisp point have to be calculated (computationally costly), it only needs to calculate Euclidean distances between pairs of crisp points.

The novel proposal is tested on six SFO problem instances derived from three real-world identification cases solved by the Physical Anthropology Lab at the University of Granada (Spain).

The structure of the paper is as follows. In Section 2 we describe the SFO problem and our previous evolutionary method to deal with it. Then, 
we study the sources of uncertainty associated with the SFO task (Section 3) and summarize our previous fuzzy approach to tackle them. Section 4 is devoted to introduce our proposal based on a cooperative coevolutionary algorithm. In Section 5, we test and compare the new proposal against the fuzzy-evolutionary one over six problem instances. Finally, we present some concluding remarks and future works in Section 6.

\section{Skull-face overlay in craniofacial superimposition}

The success of the SFO process requires positioning the skull in the same pose of the face as seen in the given photograph (provided by the relatives of the missing/deceased person). The orientation process is a very challenging and time-consuming part of the CS technique [4].

Most of the existing SFO methods are guided by a number of anthropometrical landmarks located in both the skull and the photograph of the missing person. The selected landmarks are placed in those parts where the thickness of the soft tissue is low. The goal is to ease their location when the anthropologist must deal with changes in age, weight, and facial expressions.

Once these landmarks are available, the SFO procedure is based on searching for the skull orientation leading to the best matching of the two sets of landmarks.

In view of the task to be performed, the relation of the desired procedure with the image registration (IR) problem in computer vision [9] can be clearly identified. Following a 3D-2D IR approach we aim to properly align the $3 \mathrm{D}$ skull model and the $2 \mathrm{D}$ face photograph in a common coordinate frame. The required perspective transformation to be applied on the skull was modeled in [5] as a set of geometric operations involving twelve parameters/unknowns which are encoded in a real-coded vector to represent a superimposition solution.

Formally, SFO can be formulated as follows: given two sets of $2 \mathrm{D}$ facial and $3 \mathrm{D}$ cranial landmarks, $F$ and $C$, respectively, both comprising N landmarks:

$F=\left[\begin{array}{cccc}x_{f_{1}} & y_{f_{1}} & 1 & 1 \\ x_{f_{2}} & y_{f_{2}} & 1 & 1 \\ \vdots & \vdots & \vdots & \vdots \\ x_{f_{N}} & y_{f_{N}} & 1 & 1\end{array}\right] C=\left[\begin{array}{cccc}x_{c_{1}} & y_{c_{1}} & z_{c_{1}} & 1 \\ x_{c_{2}} & y_{c_{2}} & z_{c_{2}} & 1 \\ \vdots & \vdots & \vdots & \vdots \\ x_{c_{N}} & y_{c_{N}} & z_{c_{N}} & 1\end{array}\right]$

the overlay procedure aims to solve a system of equations with the following twelve unknowns: a rotation represented by an axis $\left(d_{x}, d_{y}, d_{z}\right)$ and angle $(\theta)$, a center of mass $\left(r_{x}, r_{y}, r_{z}\right)$, a translation vector $\left(t_{x}, t_{y}, t_{z}\right)$, a uniform scaling $(s)$, and a $3 \mathrm{D}-$ $2 \mathrm{D}$ projection function given by a field of view $(\phi)$. The corresponding equation system is as follows:
$F=f(C)=C \cdot\left(A \cdot D_{1} \cdot D_{2} \cdot R_{\theta} \cdot D_{2}^{-1} \cdot D_{1}^{-1} \cdot A^{-1}\right) \cdot S \cdot T \cdot P$

where $R=\left(A \cdot D_{1} \cdot D_{2} \cdot R_{\theta} \cdot D_{2}^{-1} \cdot D_{1}^{-1} \cdot A^{-1}\right)$ represents a rotation matrix to orient the skull in the same pose of the photograph.

$S, T$, and $P$ are uniform scaling, translation, and perspective projection matrices, respectively. The interested reader is referred to [10] for a detailed description of the matrices in Equation 1 and their relation with the twelve unknowns of the problem, as well as to [5] for a deeper explanation.

Different definitions of the fitness function were studied and the one achieving the best results was the mean error $(\mathrm{ME})^{1}$ :

$$
M E=\frac{\sum_{i=1}^{N}\left\|f\left(c l^{i}\right)-f l^{i}\right\|}{N}
$$

where $\|\cdot\|$ is the $2 \mathrm{D}$ Euclidean distance, $N$ is the number of considered landmarks (provided by the forensic experts), $c l^{i}$ corresponds to every 3D craniometric landmark, $f l^{i}$ refers to every $2 \mathrm{D}$ facial landmark, $f$ is the function which defines the geometric 3D-2D projective transformation, and $f\left(c l^{i}\right)$ represents the projected skull 3D landmark $c l^{i}$ in the image/photo plane.

Solving the SFO problem in the latter fashion results in a really complex optimization task, with a highly multimodal landscape, and forensic experts demand very robust and accurate results. This complex landscape leaded us to tackle the problem considering robust evolutionary algorithms (EAs) [11] to search for the optimal values of the twelve registration transformation parameters. In [5], CMA-ES [12] and different real-coded genetic algorithms [13] were applied, achieving very promising results in some problem instances.

\section{Sources of uncertainty in skull-face overlay}

The whole CS process is influenced by uncertainty. In particular, SFO is affected by two different sources of uncertainty of different nature. On the one hand, there is an inherent uncertainty associated with the two different kinds of objects involved in the process, i.e. a skull and a face. On the other hand, there is also uncertainty associated with the $3 \mathrm{D}-2 \mathrm{D}$ overlay process that tries to superimpose a $3 \mathrm{D}$ model over a 2D image. Following, we will summarize the main characteristics of both sources and we will review our previous proposal based on the use of fuzzy landmarks to overcome most of the limitations associated with the latter sources of uncertainty.

\footnotetext{
${ }^{1}$ Notice that, mean square error is not used because of its negative effect when image ranges are normalized in $[0,1]$.
} 


\subsection{Uncertainty inherently associated with the objects under study}

We have identified two inherent sources of uncertainty regarding the handled objects (a skull and a face) and their relationship. On the one hand, the landmark location uncertainty is related to the extremely difficult task of locating the points in an invariable place, since the definition of any anthropometric landmark is imprecise in its own. Indeed, every forensic anthropologist is prone to locate the landmarks in a slightly different position [14], regardless the means used to represent the involved objects (skull and face), i.e. 3D model, 2D photo, video shot, ...

On the other hand, the landmark matching uncertainty refers to the imprecision involved in the matching of two sets of landmarks corresponding to two different objects: a face and a skull. There is a clear partial matching situation. The correspondence between facial and cranial anthropometric landmarks is not always symmetrical and perpendicular, some landmarks are located in a higher position in the alive person face than in the skull, and some others have not got a directly related landmark in the other set [2]. Besides, the facial soft tissue depth varies for each cephalometric landmark and also for every person group (regarding age, race, and sex).

\subsection{Uncertainty associated with the $3 D$ skull model-2D face photo overlay process}

The uncertainty associated with the 3D skull-2D face overlay is not inherent to the objects themselves but to our approach, where we try to overlay a 3D model and a 2D image. As done in Section 3.1, we can also distinguish between landmark matching and landmark location uncertainty. However, the nature of these two sources of uncertainty is different in the current case.

On the one hand, the landmark location uncertainty refers to the difficulty of locating landmarks with the accuracy required for the automatic overlay of a $3 \mathrm{D}$ skull model and a $2 \mathrm{D}$ face photo. The ambiguity may arise from reasons like variation in shade distribution depending on light condition during photographing, unsuitable camera focusing, poor image quality, face pose in the photograph, partial or whole landmark occlusion, etc.

Forensic experts are prone to only locate those landmarks which can be unquestionably identified in the skull and in the face. Therefore, they are usually only able to locate a reduced set of all the available cephalometric landmarks.

On the other hand, the matching uncertainty refers to the negative influence of a small number of landmarks with an unsuitable spatial distribution in the quality of the SFO results. This effect happens when the landmarks guiding the optimization method are coplanar or near-coplanar.

Due to landmark coplanarity the Equation 1 becomes undetermined (or near-undetermined), i.e. there is uncertainty (there is not enough information or it is imprecise) regarding which of the possible solutions is the best. As a consequence, it is not possible to numerically distinguish among the different resulting (after a search process) sets of projection parameters which originate different SFO results. In [15] we experimentally demonstrated the strong negative impact of coplanar landmarks in the quality of the SFO results derived by our automatic procedure. Having a reasonable number of anthropometrical landmarks located in different planes becomes a real need for the good performance of the method.

\subsection{Imprecise landmarks: a fuzzy set-based approach}

Our approach is based on allowing the forensic anthropologist to perform an imprecise location of cephalometric landmarks. By using imprecise landmarks, (s)he can locate the landmark as a region instead of as a crisp point as usual. The size of the region defined by the forensic expert will become a measure of the landmark uncertainty (the broader the region, the higher the uncertainty in the location of that landmark).

Notice that, by marking landmarks in an imprecise way, we manage to solve the problems related to three of the four uncertainty sources analyzed at the same time. First, the inherent uncertainty of the landmark location in the missing person photograph can be properly tackled. In the same way, the forensic experts are able to deal with the location of landmarks whose position they can not determine accurately due to the photograph conditions with the proper level of confidence (using imprecise regions of different sizes). As a consequence, we will allow them to deal with the extremely difficult task to increase the number of selected landmarks. As explained, those additional landmarks are essential to face the coplanarity problem in the automatic search of the best SFO. Only the landmark matching uncertainty has been left for future works.

The imprecise landmark location approach was implemented through two alternative models of imprecise landmarks: weighted and fuzzy ones. We summarize the fuzzy one since it achieved better skull-face overlay results. For a detailed description of both approaches the interested reader is referred to $[7]$.

The fuzzy landmark approach is based on allowing the forensic experts to locate the cephalometric landmarks using ellipses and on considering fuzzy sets to model the uncertainty related to them. Besides, we have also considered fuzzy distances to model the distance between each pair of craniometric and cephalometric landmarks. 
We have defined a fuzzy landmark as a fuzzy convex set of points having a nonempty core and a bounded support. That is, all its $\alpha$-levels are nonempty bounded and convex sets. In our case, since we are dealing with $2 \mathrm{D}$ photographs with an $x \times y$ resolution, we have defined the fuzzy landmarks as $2 \mathrm{D}$ masks represented as a matrix $M$ with $m_{x} \times m_{y}$ points (i.e., a discrete fuzzy set of pixels). Each fuzzy landmark will have a different size depending on the imprecision on its localization but at least one pixel (i.e. crisp point related to a matrix cell) will have membership with degree one.

These masks are easily built starting from two triangular fuzzy sets $\widetilde{V}$ and $\widetilde{H}$ modeling the approximate vertical and horizontal position of the ellipse representing the location of the landmark, thus becoming two-dimensional fuzzy sets.

An example of these fuzzy cephalometric landmarks is given in Figure 1, where the corresponding membership values (calculated using the product tnorm) of the pixels of one of those landmarks is depicted on the right.

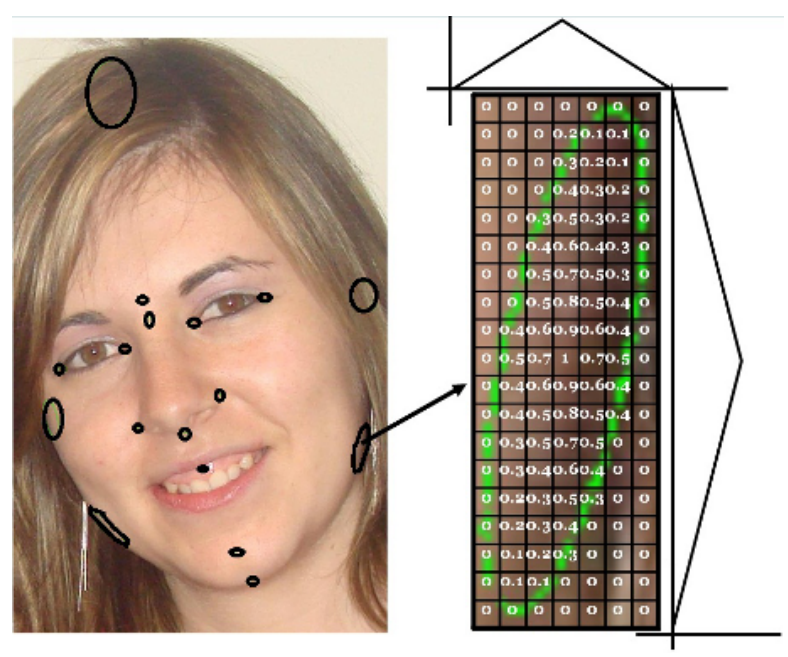

Figure 1: Example of fuzzy location of cephalometric landmarks (on the left) and representation of an imprecise landmark using fuzzy sets (on the right).

Now we can calculate the distance between a point (which will be the pixel constituting the projection of a $3 \mathrm{D}$ craniometric landmark on the $2 \mathrm{D}$ face photo) and a fuzzy landmark (the discrete fuzzy set of pixels representing the imprecise position of the cephalometric landmark).

If we denote as $d_{i}=d\left(x, \widetilde{F}_{\alpha_{i}}\right)$ the distance from point $x$ to the $\alpha$-level set $\widetilde{F}_{\alpha_{i}}$ (in this specific case, this is the minimum Euclidean distance from point $x$ to the all the points in $\alpha_{i}$ ), then the distance from the point to the fuzzy landmark $\widetilde{F}$, can be expressed by:

$$
d^{*}(x, \widetilde{F})=\frac{\sum_{i=1}^{m} d_{i} \cdot \alpha_{i}}{\sum_{i=1}^{m} \alpha_{i}}
$$

Note that the implemented distance between a point and a fuzzy set of points is quite similar to that defined in [16]. In fact, it has been already used in other image processing applications in [17].

Therefore, we modified the original definition of our evolutionary SFO technique's fitness function as follows:

$$
\text { fuzzy } M E=\frac{\sum_{i=1}^{N} d^{*}\left(f\left(c l^{i}\right), \widetilde{F}^{i}\right)}{N}
$$

where $N$ is the number of considered landmarks; $c l^{i}$ corresponds to every $3 \mathrm{D}$ craniometric landmark; $f$ is the function which defines the geometric $3 \mathrm{D}-2 \mathrm{D}$ transformation; $f\left(c l^{i}\right)$ represents the position of the transformed skull 3D landmark $c l^{i}$ in the projection plane, that is to say, a crisp point; $\widetilde{F}^{i}$ represents the fuzzy set of points of each $2 \mathrm{D}$ cephalometric landmark; and, finally, $d^{*}\left(f\left(C_{i}\right), \widetilde{F}^{i}\right)$ is the distance between a point and a fuzzy set of points.

\section{A cooperative coevolutionary algorithm tackling the landmark location uncertainty in craniofacial superimposition}

EAs have been applied to many types of problem domains, such as parameter optimization and machine learning. A very natural, and increasingly popular extension when problems domains are potentially complex, or when it is difficult or impossible to assess an objective fitness measure for the problem, is the class of so-called coevolutionary algorithms (CEAs) [8]. In such algorithms, fitness itself becomes a measurement of interacting individuals. This ostensibly allows the potential for evolving greater complexity by allowing pieces of a problem to evolve in tandem, as well as the potential for evolving solutions to problems in which such a subjective fitness may, in fact, be necessary (i.e., game playing strategies). Hence, a coevolutionary algorithm is an EA (or collection of EAs) in which the fitness of an individual depends on the relationship between that individual and other individuals [18].

Depending on the nature of the interactions among individuals we can distinguish between competitive and cooperative CEAs. In the former, each specie competes with the rest. In the latter, named cooperative coevolutionary algorithms (CCEAs) [19], all the species collaborate to build a solution for the problem. The originally-stated aim of CCEAs was to attack the problem of evolving complicated objects by explicitly breaking them into parts, evolving the parts separately, and then assembling the parts into a working whole.

This is the goal of the current proposal in which two different but complementary problems arise. On the one hand, we want to know the best set of transformation parameters resulting in the best possible SFO. On the other hand, SFO quality is 
measured based on the distances among two set of landmarks, where the location of the cephalometric one is uncertain. We only know that they are located somewhere inside a region delimited by the forensic expert (imprecise landmark). Hence, we can try to find the exact locations of the cephalometric landmarks in that region. However, the only way to determine them is by looking for the best SFO, assuming that the most precise location of the landmarks implies the chance to achieve the most precise SFO. That is to say, we need to look, at the same time, for the best set of transformation parameters and for the most precise cephalometric landmarks locations.

To do that, we have implemented a CCEA where two populations optimize the set of transformation parameters and the location of the cephalometric landmark, respectively. For the former population we consider a coding scheme representing the twelve registration transformation parameters in a vector of real numbers while for the latter we consider a variable-length integer-valued chromosome, whose size corresponds with the number of pair of landmarks used. Both populations need to collaborate/interact to construct a solution for the main problem, that is to achieve the best possible SFO.

The question of how a practitioner determines collaborators or competitors may be among the most important design decisions for the successful application of CEAs [20]. The most obvious (and computationally expensive) method to evaluate an individual in a coevolutionary setting is for an individual to interact with all possible collaborators or competitors. In the case of binary interactions, this is sometimes called complete pairwise interaction. An alternative extreme is for an individual to be involved in only a single interaction. Such a choice leaves open the obvious question of how to pick the collaborator or competitor. Of course, between these two extremes there is a whole host of possibilities that involve some subset of interactions. Again, collaborators/competitors for such interactions may be chosen in a variety of ways from uniformly random, to fitness biased methods. There are mainly three attributes of this choice, suggesting a wide range of possible strategies. They are briefly described below:

- Interaction sample size: the number of collaborators/competitors from each population to use for a given fitness evaluation.

- Interaction selective bias: the degree of bias of choosing a collaborator/ competitor. For example, an individual could be selected either randomly or based on the fitness value.

- Interaction credit assignment: the method of credit assignment of a single fitness value from multiple interaction-driven objective function results. The main examples here are the minimum, the maximum and the mean of all the fitness values.
In our implementation of the CCEA we tested many different options for these three parameters. As interaction selective bias we use both the best individuals and random ones. The minimum, the maximum and the mean were tested as credit assignment mechanisms.

The transformation parameters population uses SBX crossover [5] while the landmark locations population uses multi-point crossover. Random mutation is used in both cases, constraining the possible values for the landmark location to all the pixels inside the region corresponding to the imprecise landmark the forensic expert located in the image.

In the following section we show the set of parameters that results in the best SFO results.

\section{Experiments}

Our experimental study will involve six different SFO problem instances corresponding to three realworld cases previously addressed by the staff of the Physical Anthropology lab at the University of Granada (Spain) in collaboration with the Spanish scientific police.

All those identification cases were positively solved following a computer-supported but manual approach for SFO. We will consider the available 2D photographs of the missing people and their respective 3D skull models acquired at the lab by using its Konica-Minolta ${ }^{\circledR}$ 3D Lasserscanner VI-910.

The experiments developed in this section are devoted to study the performance of the coevolutionary-based approach to model the imprecise location of cephalometric landmarks within our SFO method in comparison with the previous fuzzy set-based proposal. With this aim, we first show the parameter setting considered. Then, we introduce each of the six selected real-world SFO problems to be tackled together with the obtained results and their analysis.

\subsection{Experimental design}

We used CMA-ES as the evolutionary algorithm for the case of fuzzy set-based approach [7] guided by the corresponding objective function (Equation 3) and we considered the following set of parameters:

$$
\begin{array}{lll}
\text { initial } \theta \text { (mutation distribution variance) } & = & 0.1 \\
\lambda(\text { population size, offspring number }) & = & 100 \\
\mu(\text { number of parents/points for recombination }) & = & 15
\end{array}
$$

The rest of the parameters are the default ones, reported in [12].

For the case of the coevolutionary approach, we performed many different combinations of parameters $^{2}$ under the restriction of 560,000 evaluations of the fitness function (according to the CMA-ES conditions). The set of parameters considered are the following:

\footnotetext{
${ }^{2}$ These preliminary experiments are not included because of a lack of space
} 


\subsection{Case studies}

$\begin{array}{lll}\text { \# generations } & =600 \\ \mathrm{~T} \text { size } & =450 \\ \mathrm{~L} \text { size } & =25 \\ \mathrm{~T} \text { and L crossover probability } & =0.9 \\ \mathrm{~T} \text { and L mutation probability } & =0.2 \\ \mathrm{~T} \text { and L tournament size (for RCGA) } & =2 \\ \text { SBX } \eta \text { parameter (for population T) } & =0.1 \\ \text { Interaction sample size and selective bias: } & \\ \text { \# best selected individuals in T } & =1 \\ \text { \# random selected individuals in T } & =1 \\ \text { \# best selected individuals in L } & =2 \\ \text { \# random selected individuals in L } & =2 \\ \text { Interaction credit assignment } & =\mathrm{m}\end{array}$

where $\mathrm{T}$ is the population of the transformation parameters, L is the population of landmark locations and $m$ corresponds to the minimum value from the multiple interaction-driven objective function results.

Thirty independent runs were performed for each case in order to avoid bias in the results and to compare the robustness of both proposals.

We would like to have a quantitative measure allowing us to benchmark the achieved outcomes. Unfortunately, the ME values obtained by each approach are not fully significant to perform a comparison because of the different objective functions to be minimized. In addition, there is not a direct correspondence between ME values and the visual representations as it was pointed out by the experts in $[7]$.

Due to the latter reasons, we adopted an alternative, specifically designed image processing scheme to evaluate the performance of every SFO approach called "area deviation error" (ADE) $[7,15]$. In ADE the percentage of the head boundary that is not covered by the area of the projected skull is computed as a measure of the quality of the overlay. Figure 2 shows an example of the application of this evaluation procedure.

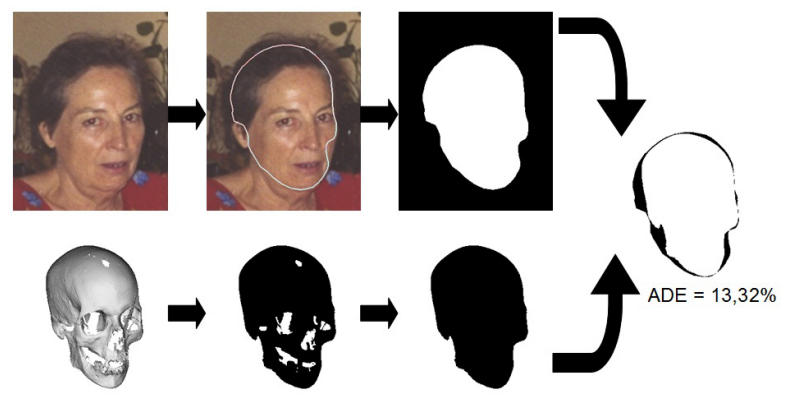

Figure 2: Example of area deviation error procedure. From left to right, original photographs (top) and projected skull (bottom), intermediate images with the head boundary (top) and binary skull (bottom), and final XOR image (right most) with the corresponding $\mathrm{ADE}$ value below the image.
As said, all the SFO instances tackled in this work are real-world cases previously addressed by the Physical Anthropology lab at the University of Granada (Spain). We briefly introduce them as follows.

The first case study happened in Málaga, Spain. The facial photograph of this missing lady was provided by the family and the final identification done by CS has been confirmed. The forensic experts manually selected a set of fifteen 2D cephalometric landmarks on the face present in the photo, following an imprecise approach (first photograph of Figure 3).
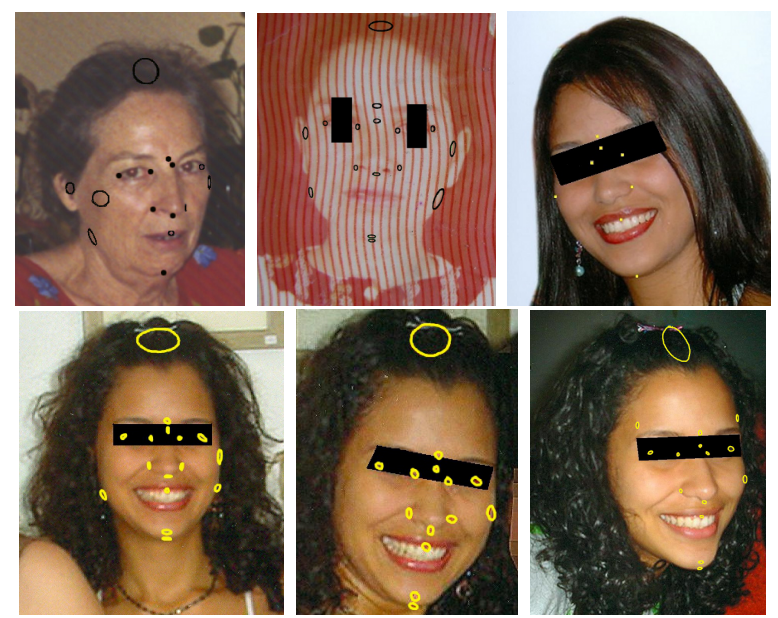

Figure 3: Case studies: photograph of the missing person with the corresponding set of imprecise landmarks. First row, first image corresponds to case study 1 . First row, second image corresponds to case study 2. The other four images (last image of first row and the three images in the second row) belong to case study 3 , poses $1,2,3$ and 4 , respectively.

The second real-world case considered corresponds to a Moroccan woman whose corpse was found in the South of Spain. There is a single available photograph corresponding to that one in the alleged passport. In this case of study, the forensic experts identified 16 cephalometric landmarks following an imprecise approach (second photograph of Figure 3).

Finally, The third case study happened in Cádiz, Spain. The four different photographs were provided by the relatives, which acquired them at different moments and in different poses and conditions. Hence, this case study consists of four distinct SFO problem instances. The forensic experts were able to locate 14, 16, 15 and 8 landmarks following an imprecise approach for poses 1, 2, 3 and 4, respectively (third to sixth photograph of Figure 3 ). 

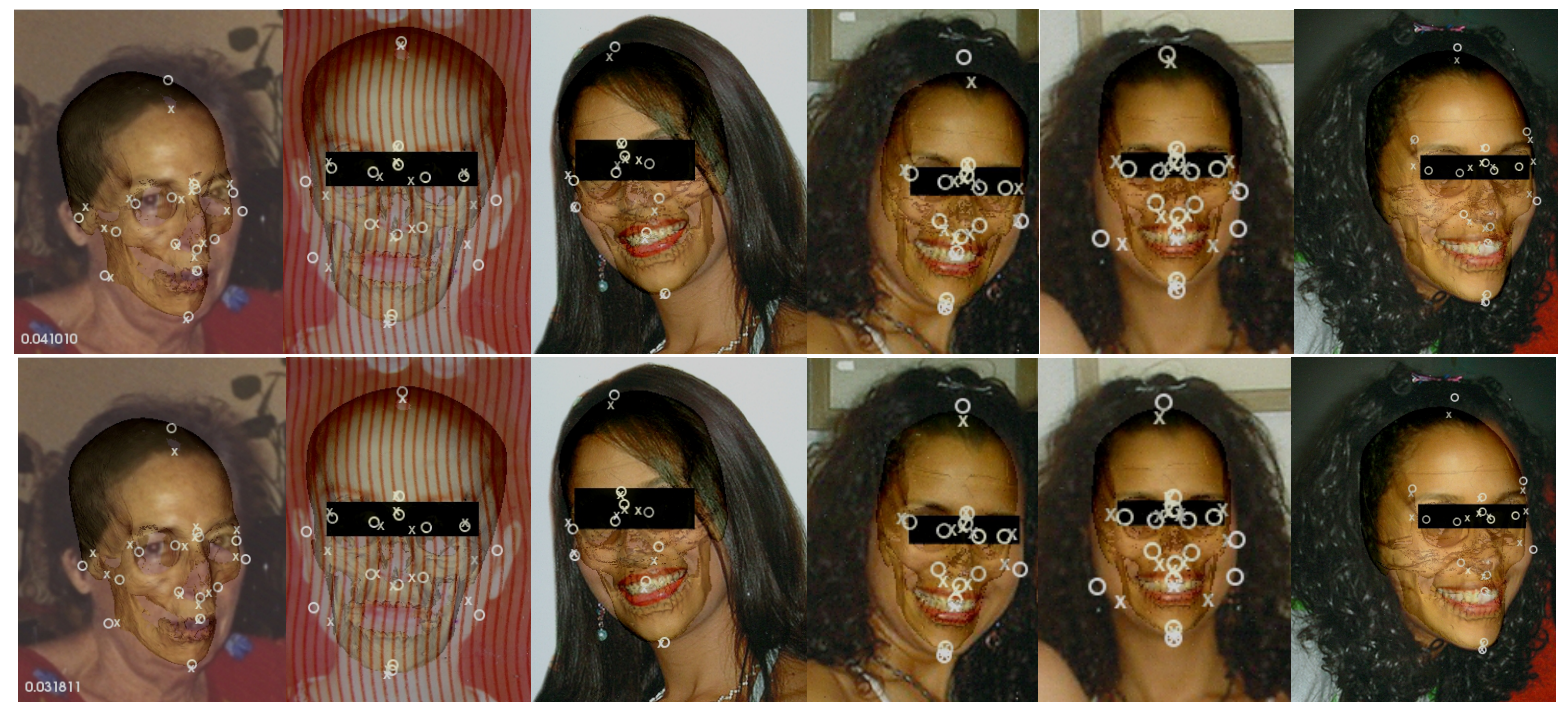

Figure 4: Best superimposition obtained following a fuzzy-evolutionary (first row) and a coevolutionary (second row) approaches. From left to right, case studies 1, 2, 3 (poses 1 to 4).

Table 1: Mean run time needed to execute the fuzzy-evolutionary and the coevolutionary approaches over each of the SFO instances.

\begin{tabular}{|c|c|c|c|c|c|c|}
\hline \multirow{2}{*}{ Approach } & \multicolumn{7}{|c|}{ Run time } \\
\cline { 2 - 7 } & case 1 & case 2 & case 3, 1 & case 3, 2 & case 3, 3 & case 3, 4 \\
\hline Fuzzy & $55^{\prime}$ & $190^{\prime}$ & $63^{\prime}$ & $82^{\prime}$ & $97^{\prime}$ & $210^{\prime}$ \\
\hline Coevolution & $1.8^{\prime}$ & $4.8^{\prime}$ & $3.9^{\prime}$ & $2.7^{\prime}$ & $3.2^{\prime}$ & 4.6 \\
\hline
\end{tabular}

\subsection{Coevolution vs fuzzy landmarks}

Table 1 shows the run time of both approaches in every SFO instance. Our coevolutionary proposal is, between, ten and thirty times faster than the fuzzyevolutionary method. As said, these big differences arise mainly because of the high computational effort needed to calculate the fuzzy distance between each pair of crisp-fuzzy landmarks in every fitness evaluation.

Table 2 presents the ADE values for the obtained SFOs in the previous six cases, distinguishing between fuzzy-evolutionary and coevolutionary approaches. The minimum (m), maximum (M), mean $(\varpi)$, and standard deviation $(\sigma)$ values of the thirty runs performed are shown for each case.

Mean and standard deviation ADE values show a better performance of the fuzzy-evolutionary approach. However, according to the minimum ADE results, the coevolutionary approach outperforms in four of the six cases. The corresponding SFO representation are depicted in Figure 4. As ADE values confirm, there are not big visual differences among the best SFO obtained following both approaches. In fact, visual differences can be mainly distinguished for those cases with bigger ADE differences, that is, cases 1 and 3 poses 3 and 4 .
Table 2: Case studies 1, 2, and 3 (poses 1, 2, 3, and 4). Area deviation error of the automatic skull-face overlays obtained following a fuzzy-evolutionary and a coevolutionary approaches.

\begin{tabular}{|c|l|c|c|c|c|}
\hline \multirow{2}{*}{ Case } & \multirow{2}{*}{ Approach } & \multicolumn{4}{|c|}{$A D E$} \\
\cline { 3 - 6 } & & $m$ & $M$ & $\varpi$ & $\sigma$ \\
\hline 1 & fuzzy & 15.20 & 45.98 & 21.56 & 10.60 \\
& coevolution & $\mathbf{1 2 . 2 0}$ & $\mathbf{2 2 . 1 6}$ & $\mathbf{1 7 . 4 4}$ & $\mathbf{2 . 7 4}$ \\
\hline 2 & fuzzy & 14.16 & $\mathbf{1 5 . 9 8}$ & $\mathbf{1 4 . 9 7}$ & $\mathbf{0 . 4 3}$ \\
& coevolution & $\mathbf{1 3 . 0 4}$ & 25.23 & 17.23 & 3.37 \\
\hline 3,1 & fuzzy & $\mathbf{1 4 . 5 4}$ & $\mathbf{2 0 . 6 5}$ & $\mathbf{1 6 . 5 0}$ & $\mathbf{1 . 1 4}$ \\
& coevolution & 15.42 & 41.92 & 30.19 & 7.11 \\
\hline 3,2 & fuzzy & 23.67 & $\mathbf{2 6 . 0 7}$ & $\mathbf{2 4 . 7 8}$ & $\mathbf{0 . 5 7}$ \\
& coevolution & $\mathbf{2 3 . 5 9}$ & 37.71 & 31.24 & 3.84 \\
\hline 3,3 & fuzzy & 17.32 & $\mathbf{2 0 . 6 2}$ & $\mathbf{1 8 . 9 7}$ & $\mathbf{0 . 8 8}$ \\
& coevolution & $\mathbf{1 4 . 2 2}$ & 38.55 & 26.57 & 6.58 \\
\hline 3,4 & fuzzy & $\mathbf{1 6 . 7 0}$ & $\mathbf{1 8 . 1 4}$ & $\mathbf{1 7 . 3 8}$ & $\mathbf{0 . 3 6}$ \\
& coevolution & 19.14 & 34.36 & 28.22 & 3.37 \\
\hline
\end{tabular}

\section{Concluding remarks and future works}

In this paper we have proposed a coevolutionary approach to deal with imprecise cephalometric landmarks in the SFO process. By using a CCEA we are able to look for both the best transformation parameters and the best landmark locations at the same time. 
Our proposal has been tested on six complex real-world identification cases and it has been compared with our previous fuzzy-evolutionary method. CCEA is a promising approach for SFO since it achieves outstanding results in very short time (between ten and thirty times faster than our previous method). Furthermore, the best coevolutionary results are better than the fuzzy-evolutionary ones in four of the six problem instances.

However, the robustness of the method should be increased. With that aim, we are studying new ways of interaction between the two populations.

\section{Acknowledgments}

This work is supported by the Spanish Ministerio de Educación y Ciencia (ref. TIN2009-07727), including EDRF fundings. We would like to acknowledge all the team of the Physical Anthropology lab at the University of Granada (headed by Dr. Botella and Dr. Alemán) for their support during the data acquisition and validation processes. Part of the experiments related to this work was supported by the computing resources at the Supercomputing Center of Galicia (CESGA), Spain.

\section{References}

[1] W. M. Krogman and M. Y. Iscan. The human skeleton in forensic medicine. Charles C. Thomas, Springfield, IL, 1986. 2nd edition.

[2] M. Y. Iscan. Introduction to techniques for photographic comparison. In M. Y. Iscan and R. Helmer, editors, Forensic Analysis of the Skull, pages 57-90. Wiley, 1993.

[3] C. N. Stephan. Craniofacial identification: techniques of facial approximation and craniofacial superimposition. In S. Blau and D. H. Ubelaker, editors, Handbook of Forensic Anthropology and Archaeology, pages 304-321. Left Coast Press, California, USA, 2009.

[4] T. W. Fenton, A. N. Heard, and N. J. Sauer. Skull-photo superimposition and border deaths: identification through exclusion and the failure to exclude. Journal of Forensic Sciences, 53(1):34-40, 2008.

[5] O. Ibáñez, L. Ballerini, O. Cordón, S. Damas, and J. Santamaría. An experimental study on the applicability of evolutionary algorithms to craniofacial superimposition in forensic identification. Information Sciences, 179:3998-4028, 2009.

[6] O. Ibáñez, O. Cordón, S. Damas, and J. Santamaría. A new approach to fuzzy location of cephalometric landmarks in craniofacial superimposition. In Proc. of the International Fuzzy Systems Association - European Society for Fuzzy Logic and Technologies (IFSAEUSFLAT) World Congress, pages 195-200, Lisbon, Portugal, 2009.
[7] O. Ibáñez, O. Cordón, S. Damas, and J. Santamaría. Modeling the skull-face overlay uncertainty using fuzzy sets. Technical Report AFE 2010-06, European Centre for Soft Computing, Mieres, Spain, 2010. Submitted.

[8] J. Paredis. Artificial Life, 2:355-375, 1995.

[9] B. Zitová and J. Flusser. Image registration methods: a survey. Image and Vision Computing, 21:977-1000, 2003.

[10] D. Hearn and M. Pauline Baker. Computer graphics (2nd ed.): C version. Prentice-Hall, Inc., Upper Saddle River, NJ, USA, 1997.

[11] T. Bäck. Evolutionary Algorithms in Theory and Practice: Evolution Strategies, Evolutionary Programming, Genetic Algorithms. Oxford University Press, 1996.

[12] N. Hansen and A. Ostermeier. Adapting arbitrary normal mutation distributions in evolution strategies: The covariance matrix adaptation. In Proceedings of the 1996 IEEE International Conference on Evolutionary Computation, pages 312-317, Piscataway, New Jersey, 1996.

[13] F. Herrera, M. Lozano, and J. L. Verdegay. Tackling real-coded genetic algorithms: operators and tools for the behavioural analysis. Artificial Intelligence Reviews, 12(4):265-319, 1998.

[14] J. Richtsmeier, C. Paik, P. Elfert, T.M. Cole, and F. Dahlman. Precision, repeatability and validation of the localization of cranial landmarks using computed tomography scans. The Cleft Palate-Craniofacial Journal, 32(3):217227, 1995.

[15] J. Santamaría, O. Cordón, S. Damas, and O. Ibáñez. Tackling the coplanarity problem in $3 \mathrm{D}$ camera calibration by means of fuzzy landmarks: a performance study in forensic craniofacial superimposition. In IEEE International Conference on Computer Vision, pages 16861693, Kyoto, Japan, 2009.

[16] D. Dubois and H. Prade. On distance between fuzzy points and their use for pausible reasoning. In International Conference on Systems, Man and Cybernetics, pages 300-303, 1983.

[17] I. Bloch. On fuzzy distances and their use in image processing under imprecision. Pattern Recognition, 32:1873-1895, 1999.

[18] R. P. Wiegand. An Analysis of Cooperative Coevolutionary Algorithms. PhD thesis, George Mason University, Fairfax, Virginia, 2003.

[19] M. A. Potter and K. A. De Jong. Evolutionary Computation, 8(1):1-29, 2000.

[20] Liles W. Wiegand, R. P. and K. De Jong. An empirical analysis of collaboration methods in cooperative coevolutionary algorithms. See Spector, Goodman, Wu, Langdon, Voigt, Gen, Sen, Dorigo, Pezeshk, Garzon, and Burke, pages 1235-1242, 2001. 